\title{
LAS INTERACCIONES ATMÓSFERA-MAR EN LA CLIMATOLOGÍA MEDITERRÁNEA DEL 2001
}

\author{
José Quereda Sala \\ Enrique Montón Chiva \\ José Escrig Barberá \\ Estación Climatología Aplicada \\ Universitat Jaume I (Castellón)
}

\section{RESUMEN}

En el presente trabajo se analizan las distintas situaciones atmosféricas que han afectado al mediterráneo español durante al 2001. A lo largo del mismo, las observaciones meteorológicas efectuadas en el excepcional emplazamiento del observatorio marino de la Universitat Jaume I han permitido verificar que la cuenca occidental del Mediterráneo aparece cada vez más como una zona neurálgica por lo que concierne a las interacciones atmósfera-mar. Las distintas situaciones atmosféricas observadas durante este año han mostrado que tanto las largas situaciones de estabilidad atmosférica como especialmente la génesis y evolución de sus grandes perturbaciones meteorológicas han venido determinadas por el desarrollo más o menos intenso de los procesos de termoconvección que se han generado en el interior de esa cubeta marina. Asimismo, toda esa serie de observaciones, mediante el recurso a la imagineria de satélites orbitales y a la topografía sinóptica, sugieren que tanto el mismo drenaje del aire frío hacia nuestro ámbito como la misma intensificación de los torbellinos ciclogenéticos podrían estar siendo activados por las anomalías registradas en el campo térmico del mar.

Palabras clave: interacciones atmósfera-mar, ciclogénesis, convección.

\section{ABSTRACT}

In the present work the different atmospheric situations that have affected to the Spanish Mediterranean during the year 2001 are analyzed. Throughout the same one, the conducted meteorological observations in the exceptional location of the marine observatory of the Universitat Jaume I have allowed to verify that the western basin of the Mediterranean appears more and more as a neuralgic zone reason why concerns the atmosphere-sea interactions. The different observed atmospheric situations during this year have shown that as much the long situations of atmospheric stability like specially the genesis and evolution of their great meteorological disturbances have come determined by the more or less intense development from the termal convection processes which 
they have been generated inside that marine bucket. Also, all that series of observations, by means of the resource to images of orbital satellites and the synoptic topography, as much suggests the same drainage of the cold air towards our scope as the same intensification of the cyclogenetic eddies could be being activated by the anomalies registered in the thermal field of sea.

Key words: atmosphere-sea interactions, cyclogenesis, convection.

\section{Introducción}

La cuenca occidental del Mediterráneo constituye una zona neurálgica por lo que concierne a las interacciones atmósfera-mar. En efecto, tanto las largas situaciones de estabilidad atmosférica como especialmente la génesis y evolución de sus grandes perturbaciones meteorológicas vienen determinadas por la inhibición o por el desarrollo más o menos intenso de los procesos convectivos que se generan en el interior de esa cubeta marina (figura 1). En su fondo, un mar notablemente cálido durante el otoño e invierno

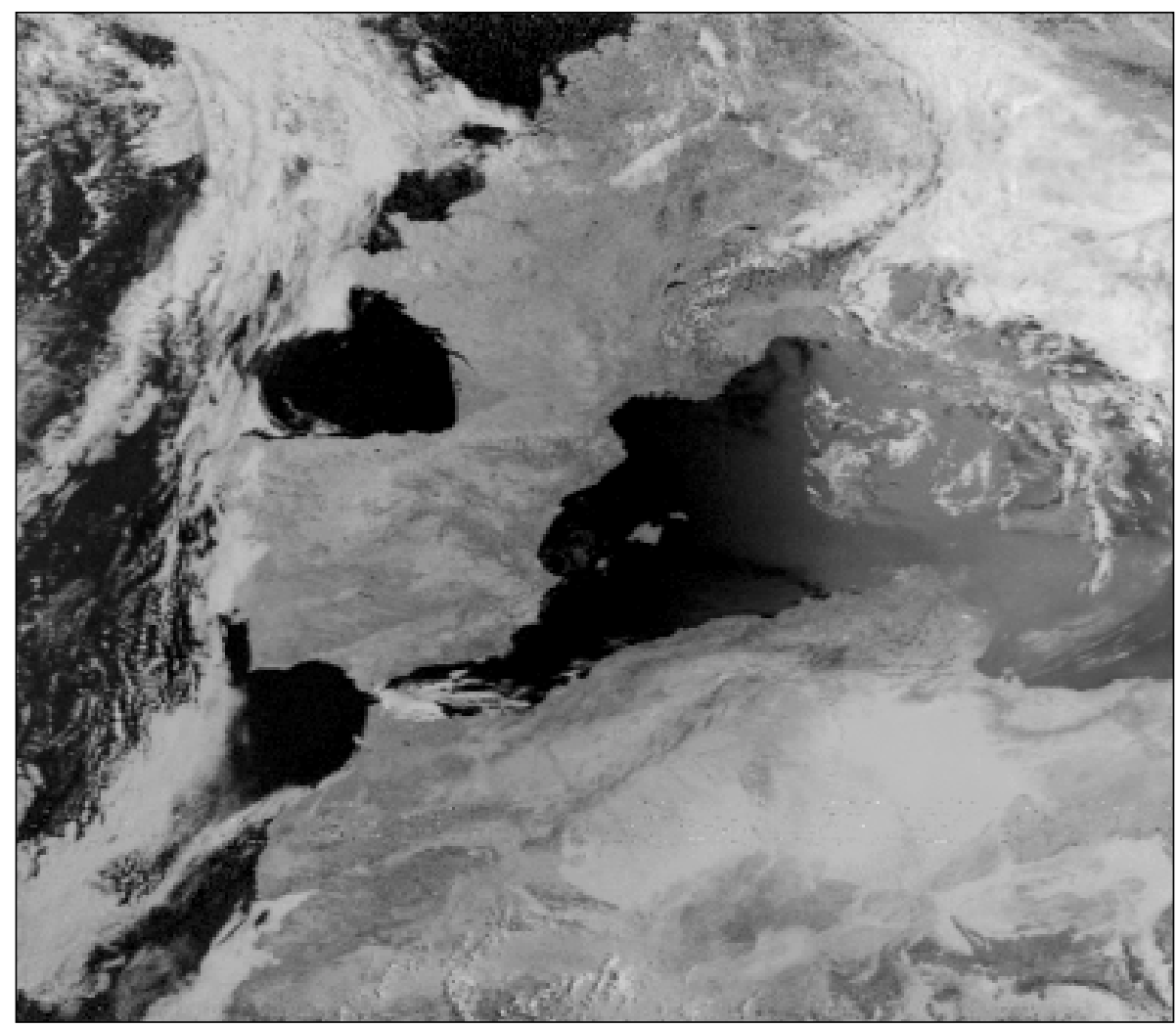

FIGURA 1. Las condiciones térmicas durante el verano con un mar refrigerante acoplan el conjunto atmósfera-mar jugando un papel decisivo en la estabilidad del clima. Estas condiciones de estabilidad, dominadas por la masa de aire tropical quedan reflejadas en los frecuentes aerosoles africanos desbordando sobre el Mediterráneo (Imagen NOAA-15, 29 de junio de 2001, canal visible, Estación de Climatología, Universitat Jaume I). 


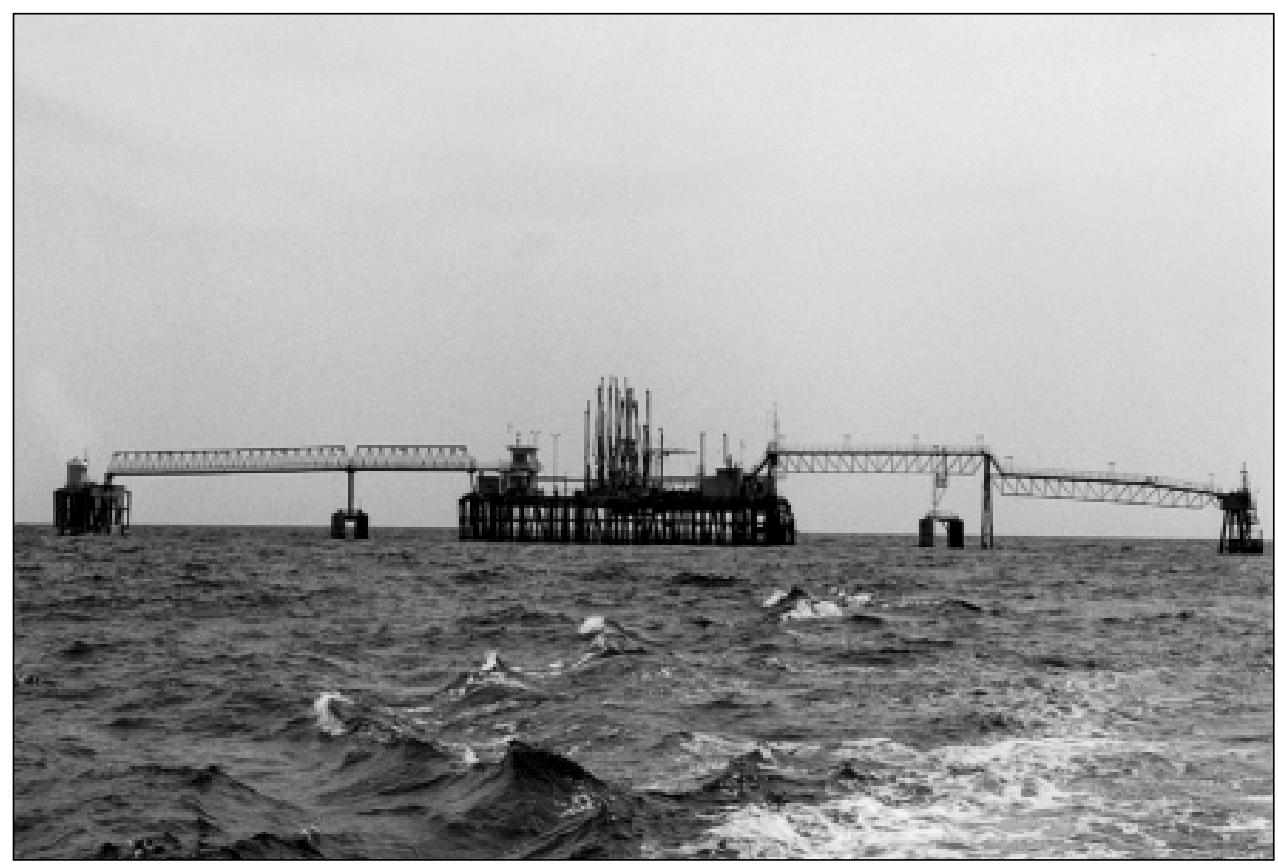

FIGURA 2. El observatorio meteorológico marino de la Universitat Jaume I en el extremo este, izquierda de la foto, sobre la Plataforma de BPOil.

constituye un manantial caliente para la masa atmosférica, mientras que en primavera y verano su función refrigeradora contiene e inhibe la convección. Este campo térmico va a ser decisivo para unos intercambios energéticos claves en la climatología mediterránea.

Consecuentemente, las observaciones meteorológicas marinas presentan un notable interés para la región mediterránea. Sin embargo ese notable interés de las observaciones marinas ha venido acompañado siempre por la dificultad de su realización. El difícil mantenimiento e instalación de observatorios dentro de mares y océanos, ha trocado en escasas las observaciones de los procesos atmosféricos desarrollados en la interfase atmósfera-mar que son claves para el diagnóstico del tiempo. Puntos fijos, boyas ancladas y derivantes, barcos colaboradores y más recientemente los satélites meteorológicos tratan de cubrir esta gran necesidad observacional.

Tal es la importancia de observatorios marinos como el de la Universitat Jaume I. Este observatorio está situado sobre la plataforma petrolífera de BPOil a $3 \mathrm{Km}$ de la costa y sobre isobatas de -15 a -20 metros (figura 2). Sus coordenadas geográficas son $39^{\circ} 56^{\prime} 42^{\prime \prime}$ $\mathrm{N}$ y $00^{\circ} 01^{\prime} 36^{\prime \prime} \mathrm{E}$. En funcionamiento convencional y semiautomatizado desde 1982, a mediados del año 2000 fue completamente renovado y automatizado en sus registros. De ahí el interés que presentan los registros de los dos primeros años de observaciones automatizadas, 2000-2001, y su relación con las situaciones atmosféricas acontecidas y cuya publicación permite, a los investigadores de la Universitat Jaume I, reiterar su enorme gratitud a BPOil. 


\section{Las interacciones atmósfera-mar}

\subsection{La radiación incidente}

La cuenca occidental del Mediterráneo aparece cada vez más como una zona neurálgica de las interacciones atmósfera-mar. Interacciones basadas en las transferencias energéticas que se operan en la interfase a partir de la radiación incidente. Es decir, de la energía subsistente después de haber atravesado los distintos filtros atmosféricos y cuyo efecto se intensifica en mares cerrados como el Mediterráneo.

La figura 3 muestra un esquema del flujo energético a través de la atmósfera. Este flujo aporta aproximadamente un valor medio de $340 \mathrm{Wm}^{-2}$ en el límite superior de la atmósfera. A partir de aquí, y a medida que penetra en capas aéreas más densas, la radiación solar incidente va a experimentar los procesos inherentes a su trayectoria a través de la atmósfera: absorción, reflexión y difusión. Ello determina que a la superficie del globo alcance un promedio del $47 \%$ (38\% en directo y $9 \%$ en difuso) de la energía solar incidente en las capas superiores de la atmósfera (C. Sinolecka et M. Hontarrède, 2000). Esta energía que ha conseguido alcanzar el globo, incide sobre una superficie que en casi un $72 \%$ está constituida por las aguas oceánicas y marinas, a cuyas capas superficiales aporta un aumento térmico.

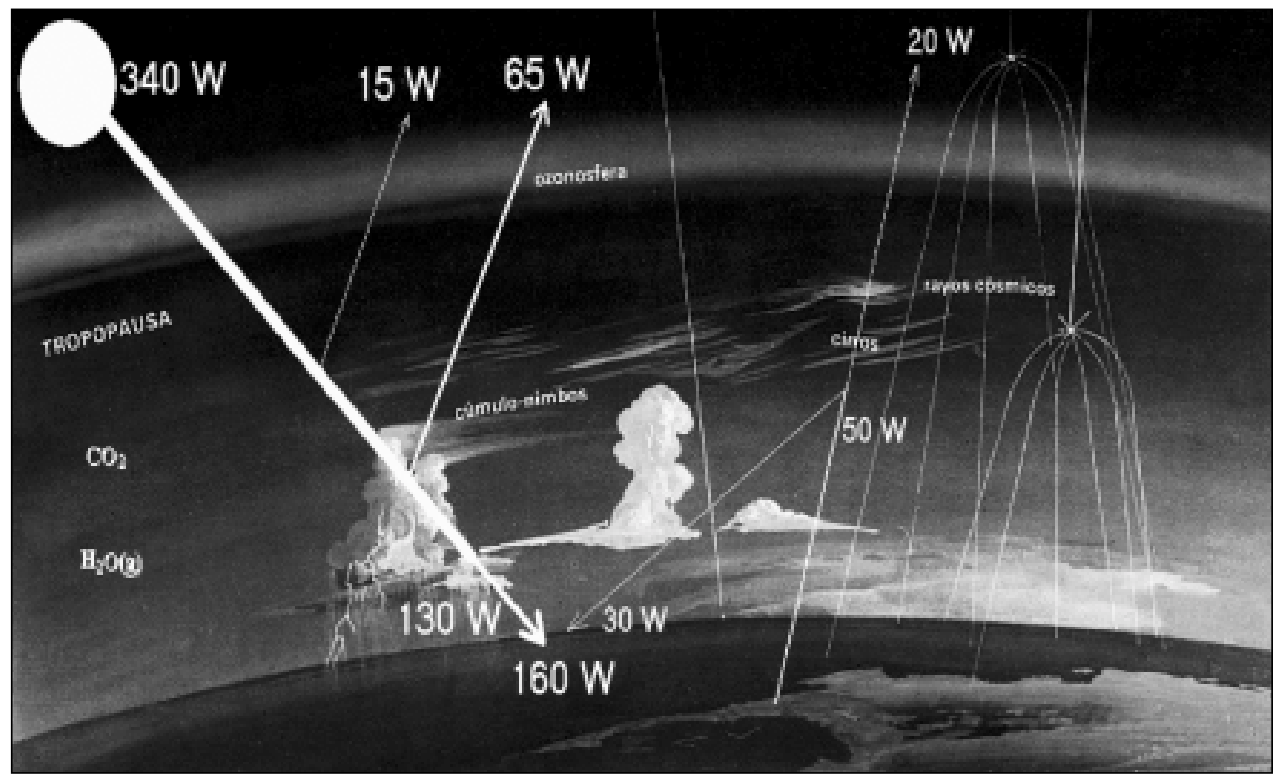

FIGURA 3. Diagrama atmosférico mostrando los distintos flujos del balance de energía solar incidente sobre la superficie marina a partir de los $340 \mathrm{Wm}^{-2}$ radiados por el sol en la cima de la atmósfera.

Cuando esa radiación residual, el $47 \%$ de la total recibida en el límite exterior de la atmósfera, alcanza la interfase aire-mar, es parcialmente reflejada y parcialmente refractada en función de la altura del sol, de la nubosidad, y del estado dinámico y óptico del mar. La superficie del mar refleja muy poco, a menos que el ángulo de incidencia de los rayos del sol sea pequeño. De ese modo, el albedo de una superficie de agua en calma es sólo del 


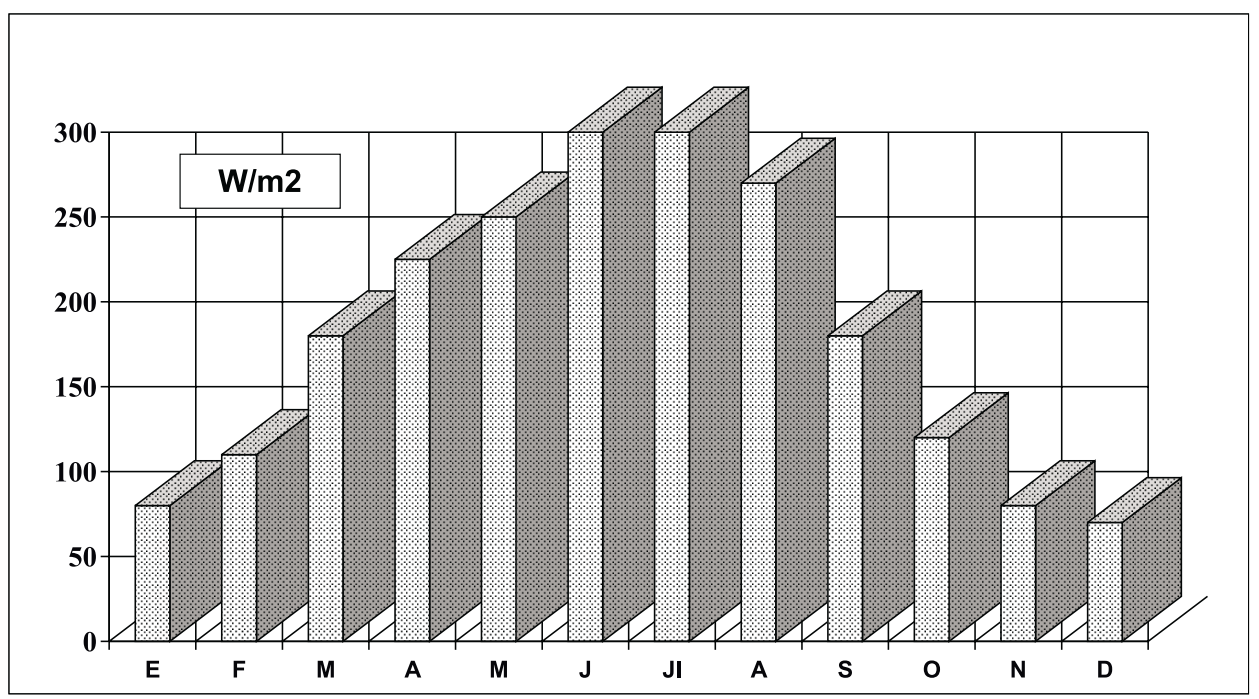

FIGURA 4. Régimen anual de la radiación solar en el observatorio marino, promedios de máximas y medias mensuales.

2 al $3 \%$ para un ángulo de elevación solar que exceda de $60^{\circ}$, pero es más del $50 \%$ cuando el ángulo es de $15^{\circ}$. Con ello se estima que, en promedio, la cantidad de energía solar que penetra en las aguas es del orden de $160 \mathrm{~W} / \mathrm{m}^{2}$ ó $0.23 \mathrm{cal} / \mathrm{cm}^{2} / \mathrm{min}$, algo más de la décima parte de la constante solar.

Las observaciones directas realizadas en el observatorio marino han permitido verificar y cuantificar estos procesos. Sobre las aguas superficiales del Mediterráneo se han registrado altos valores de radiación solar incidente. Estos valores alcanzan unos promedios mensuales máximos próximos a $300 \mathrm{~W} / \mathrm{m}^{2}\left(0.43 \mathrm{cal} / \mathrm{cm}^{2} / \mathrm{min}\right)$. El régimen anual de la radiación solar aparece en la figura 4 . Los valores máximos se alcanzan en los meses de junio y julio y los mínimos en diciembre y enero, en pleno paralelismo astronómico, con valores de tan sólo 70 a $80 \mathrm{~W} / \mathrm{m}^{2}$, equivalentes a $0.10 \mathrm{cal} / \mathrm{cm}^{2} / \mathrm{min}$.

Ello supone un valor medio anual de radiación incidente de $0.26 \mathrm{cal} / \mathrm{cm}^{2} / \mathrm{min}$. Valor muy semejante a las $0.25 \mathrm{cal} / \mathrm{cm}^{2} / \mathrm{min}$ que los cálculos teóricos (Ch. Perrin de Brichambault, 1978) y registros actinográficos dan para Castellón (J. Quereda, 1982). Es decir, un valor muy próximo al estimado como promedio global y del que en un $75 \%$ corresponde al flujo solar neto de corta longitud de onda.

Esta coincidencia con los parámetros solares se mantiene en el mismo régimen diario. Los valores máximos se registran en el cenit del día y, por el contrario, durante el periodo nocturno, lógicamente, no existe radiación solar incidente. Estos valores máximos se elevan en promedio diario estival hasta casi $1.000 \mathrm{~W} / \mathrm{m}^{2}$, equivalentes a $1.4 \mathrm{cal} / \mathrm{cm}^{2} / \mathrm{min}$, nada menos que un $75 \%$ de la constante solar. En invierno estos valores descienden, si bien conservando altas tasas de radiación solar en los momentos centrales del día. Así, los valores promedios máximos registrados en el mes de febrero se sitúan en $400 \mathrm{~W} / \mathrm{m}^{2}, 0.57$ $\mathrm{cal} / \mathrm{cm}^{2} / \mathrm{min}$ (figura 5). 


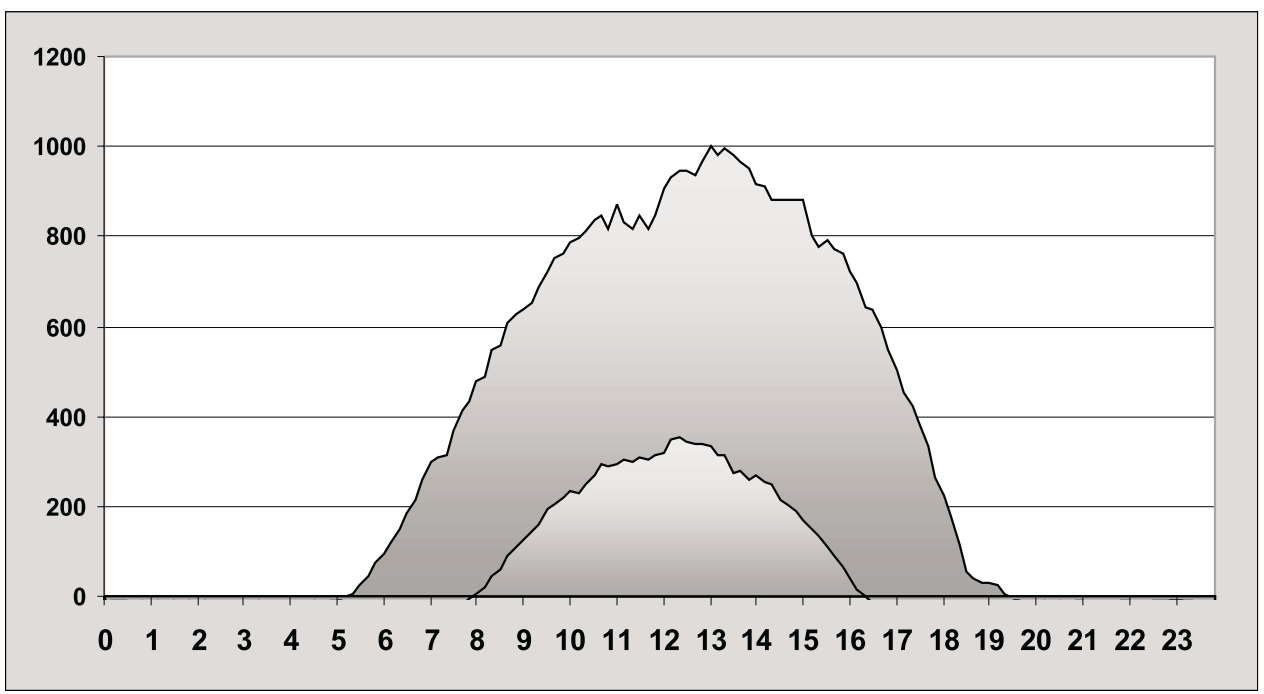

FIGURA 5. Régimen diario de la radiación solar en los meses de agosto y febrero.

De este modo la radiación incidente sobre las aguas mediterráneas es en promedio de $0.26 \mathrm{cal} / \mathrm{cm}^{2} / \mathrm{min}$. Este flujo de radiación incidente, un $12 \%$ de la constante solar, va a ser refractada. La radiación refractada en la capa líquida va rápidamente a experimentar los mecanismos de absorción y de difusión. Éstos dependen muy fuertemente de la concentración y de la naturaleza de las partículas orgánicas o minerales en suspensión del agua del mar. En definitiva, la mayor o menor facilidad que tiene el agua para absorber el calor recibido depende también de su transparencia. A partir de 1 metro de profundidad tan sólo penetra la radiación visible que en una fracción minúscula, centrada en el $0.47 \mu \mathrm{m}$ de longitud máxima de emisión, alcanzará hasta los 100 metros de profundidad (figura 6).

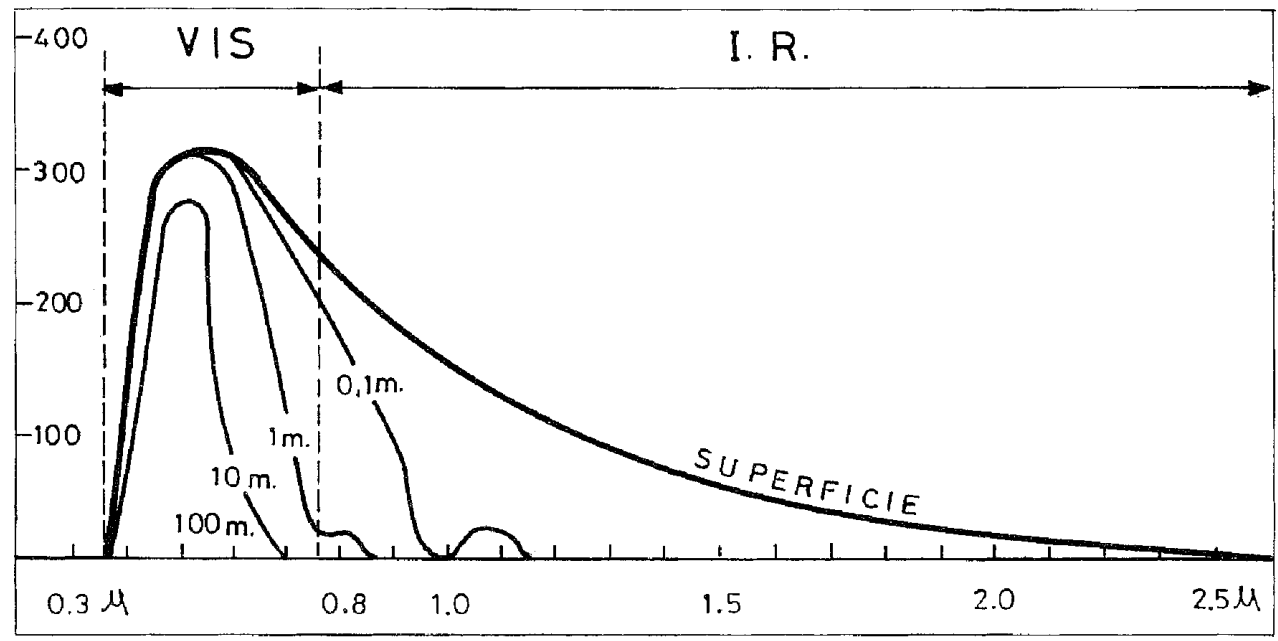

FIGURA 6. Absorción de la energía solar incidente sobre la superficie marina. 


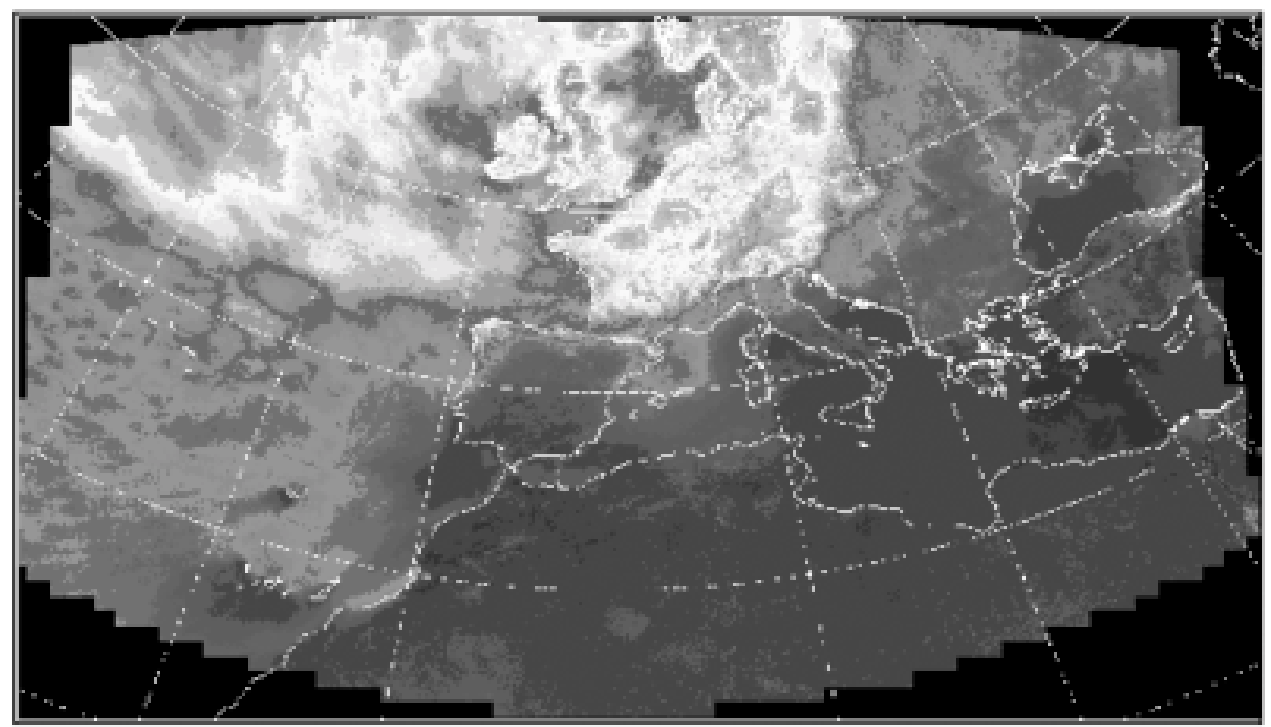

Puissance en watts par netre carre (noyenne sur 10 jours)

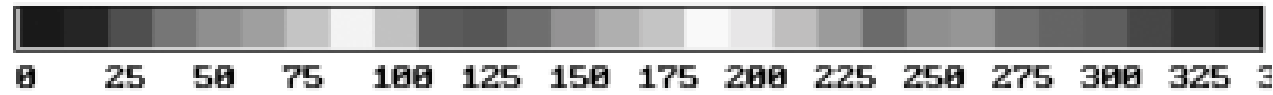

Figura 7. Potencia de radiación $\left(\mathrm{Wm}^{-2}\right)$ sobre el Mediterráneo en el mes de julio de 2001. SATMOS, Centre de Météorologie Spatiale, METEO-FRANCE.

En el ámbito del Mediterráneo Occidental, sobre el paralelo $40^{\circ} \mathrm{N}$, el balance energético tiene en su flujo positivo las $0.26 \mathrm{cal} / \mathrm{cm}^{2} / \mathrm{min}$ subsistentes tras atravesar los filtros atmosféricos y que, como Radiación Global (directa y difusa) de onda corta, alcanza la interfase atmósfera-mar (figura 7). Esta Radiación incidente $\left(\mathrm{R}_{\mathrm{i}}\right)$ es así el superavit de energía, $Q_{r}$, que incide y se refracta en la capa líquida y va a experimentar los mecanismos de absorción y de difusión.

En definitiva, este excedente de radiación, $\mathrm{Q}_{\mathrm{r}}$, representa la cantidad de energía que utiliza la columna oceánica por unidad de tiempo y de área. De tal modo que $Q_{r}=Q_{h}+Q_{e}$ $+\mathrm{Q}_{\mathrm{v}}$, es decir, que el flujo neto incidente es igual a la energía convectiva más la de evaporación más la de la entalpía propia del océano, siendo esta última, en promedio anual y en régimen estacionario, próxima a cero. Así pues, $\mathrm{Q}_{\mathrm{h}}$ y $\mathrm{Q}_{\mathrm{e}}$ representan las cantidades de energía que los torbellinos transportan verticalmente hacia la superficie marina para los procesos convectivos y los de evaporación respectivamente (figura 8). La cantidad de energía que ambos representan, $Q_{a}=Q_{h}+Q_{e}$, puede calcularse con ciertas dificultades a través de diversas fórmulas como las de Gehrke o la de Timofeev (V. K. Sukhovei and M. A. M. Said, 1985). Sin embargo, subsiste en todas ellas la dificultad, no sólo de los datos térmicos e higrométricos de la temperatura superficial del mar sino especialmente la determinación de las capas oceánicas de intercambio y la profundidad de la termoclina precisa (J. Quereda, 1986). Datos de excepcional interés para introducir el parámetro básico de las fórmulas, que es el cociente de conductividad a través de la ecuación de Fourier (J. Quereda, 1982). 


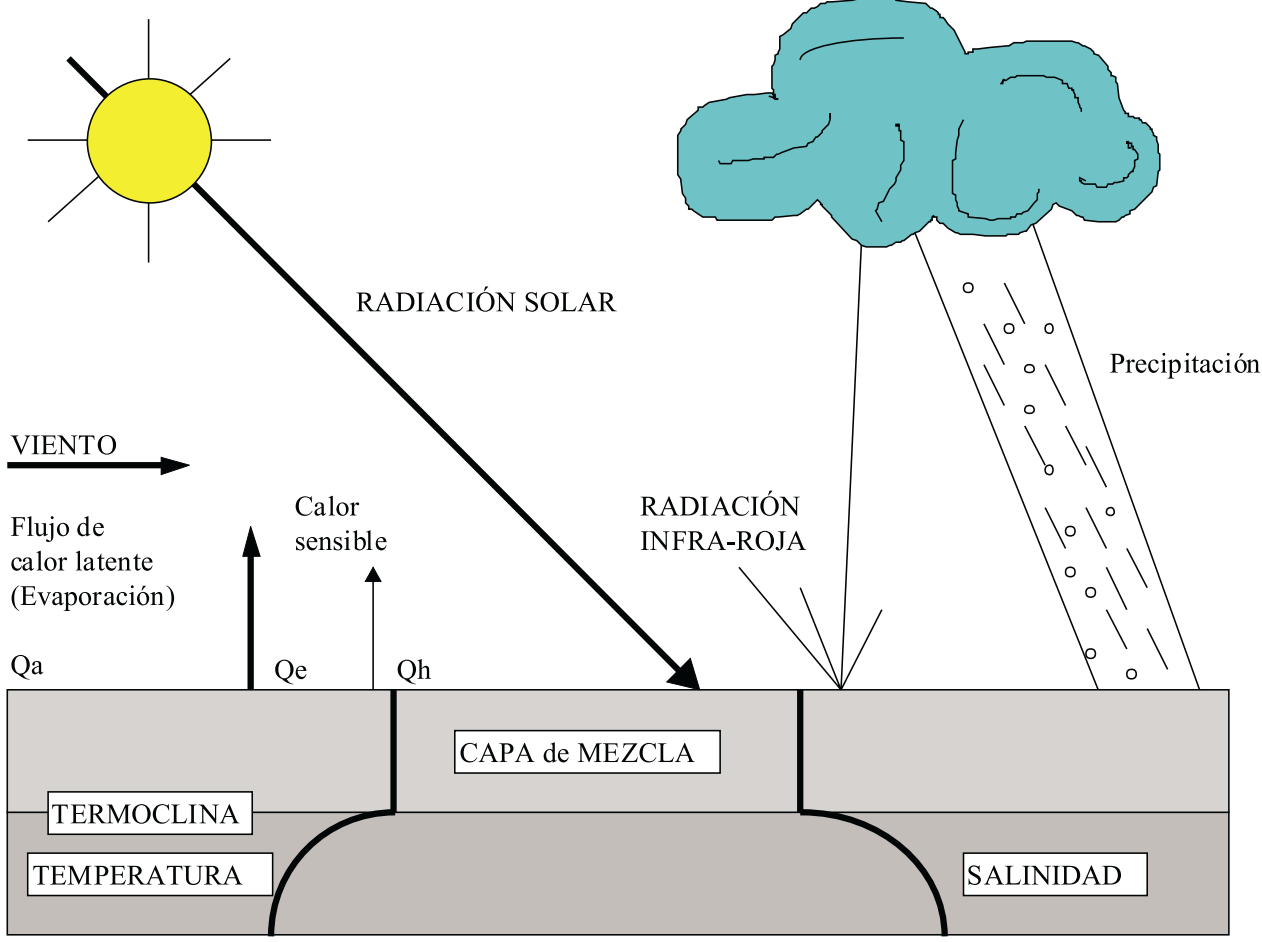

FIGURA 8. Procesos energéticos en la interfase atmósfera-mar.

\subsection{La formación del campo térmico superficial del mar}

La formación de esta capa de mezcla o capa marina activa de los intercambios con la atmósfera está condicionada, a lo largo de las diferentes estaciones del año, por diversos mecanismos. Así, durante el invierno, en nuestras latitudes, la temperatura del agua es más elevada que la del aire, lo cual origina una intensa emisión de calor desde el mar hacia la atmósfera. El enfriamiento de la Tsm origina subsiguientemente una convección libre en las aguas marinas y la subsidencia de las aguas superficiales enfriadas. En consecuencia, la mezcla convectiva incrementa el espesor de la capa casi homogénea superior y activa, que alcanza gran profundidad hacia fines del invierno. Por el contrario, durante el verano, la intensa radiación y calentamiento de las aguas marinas superficiales, cuya temperatura es muy semejante a la del aire, genera un cálido y delgado nivel de mezcla separado de las aguas subyacentes por la termoclina. Puede asumirse que el nivel total de mezcla finaliza cuando el gradiente térmico medio es de $-0.05^{\circ} \mathrm{C} / \mathrm{m}$ (Y. D. Resnyanskii, 1985) (figura 9).

Con estos datos, el valor de $\mathrm{Q}_{\mathrm{a}}$, o calor convectivo y de evaporación, transportado hasta la superficie, se puede hallar mediante una ecuación que desagregue el valor de Qe a través de una ecuación de agregación lineal:

$$
\mathrm{Q}_{\mathrm{a}}=\mathrm{Q}_{\mathrm{h}}+\mathrm{Q}_{\mathrm{e}}
$$




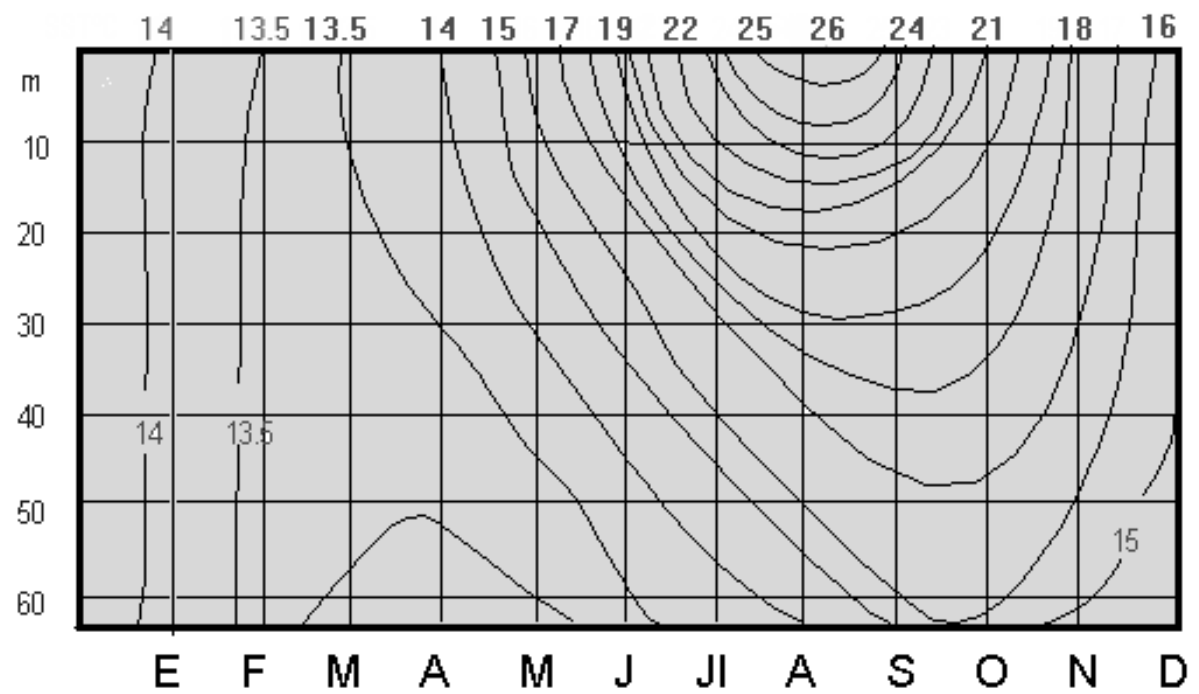

FIGURA 9. Isotermas de la capa activa en las aguas costeras mediterráneas.

A partir de esta función, el cálculo de $\mathrm{Q}_{\mathrm{e}}$, es decir, de la energía que remontada por los torbellinos hacia la superficie marina se emplea en evaporación, se logra mediante la ecuación:

$$
\mathrm{Q}_{\mathrm{e}}=6.5\left(\mathrm{e}_{\mathrm{s}}-\mathrm{e}\right) \mathrm{u}
$$

y donde $\mathrm{e}_{\mathrm{s}}$ y e representan las tensiones de vapor en superficie y a $15 \mathrm{~m}$ de altura respectivamente expresadas en milímetros, y u la velocidad media del viento en $\mathrm{m} / \mathrm{s}$. Parámetros hoy de fácil obtención y procesamiento en las estaciones automáticas de meteorología.

Los registros del observatorio marino han permitido conocer la energía $\mathrm{Q}_{\mathrm{e}}$ empleada en el proceso de evaporación. Este proceso ha dado un consumo de $0.116 \mathrm{cal} / \mathrm{cm}^{2} / \mathrm{min}$. Un valor muy próximo al que resulta de aplicar las $59 \mathrm{cal} / \mathrm{cm}^{2}$ que se precisan para evaporar un milímetro de agua (585 calorías por gramo de agua evaporada) a las cantidades evaporadas en Castellón. Los registros experimentales del Piche y tanques (J. Quereda y A. Calvo, 1981, J. Quereda et al., 2001) así como los cálculos a través de las fórmulas radioactivas de Penman (P. Brochet et N. Gerbier, 1975), dan una evaporación media diaria de $2.8 \mathrm{~mm}$; ello arroja un valor de $0.114 \mathrm{cal} / \mathrm{cm}^{2} / \mathrm{min}$, de energía utilizada en las evaporaciones, valor realizado sobre la superficie marina.

De este modo, a partir de $\mathrm{Q}_{\mathrm{e}}$ es posible alcanzar el valor de $\mathrm{Q}_{\mathrm{h}} \mathrm{o}$ energía convectiva, calor sensible empleado en calentar el aire, mediante la ecuación $\mathrm{Q}_{\mathrm{h}}=4.3\left(\mathrm{t}_{\mathrm{s}}-\mathrm{t}\right) \mathrm{u}$, habiendo aproximado las temperaturas potenciales a las correspondientes temperaturas (J. A. Naya, 1984). Aplicada la ecuación al ámbito marino de Castellón, y aproximando, da un valor medio de $0.023 \mathrm{cal} / \mathrm{cm}^{2} / \mathrm{min}$. Con ello ya es posible estimar el calor que es transmitido por la masa marina a la atmósfera $\mathrm{Q}_{\mathrm{a}}=\mathrm{Q}_{\mathrm{h}}+\mathrm{Q}_{\mathrm{e}}$. Los cálculos realizados sobre el ámbito marítimo de Castellón dan los resultados medios reflejados en la figura 10: 


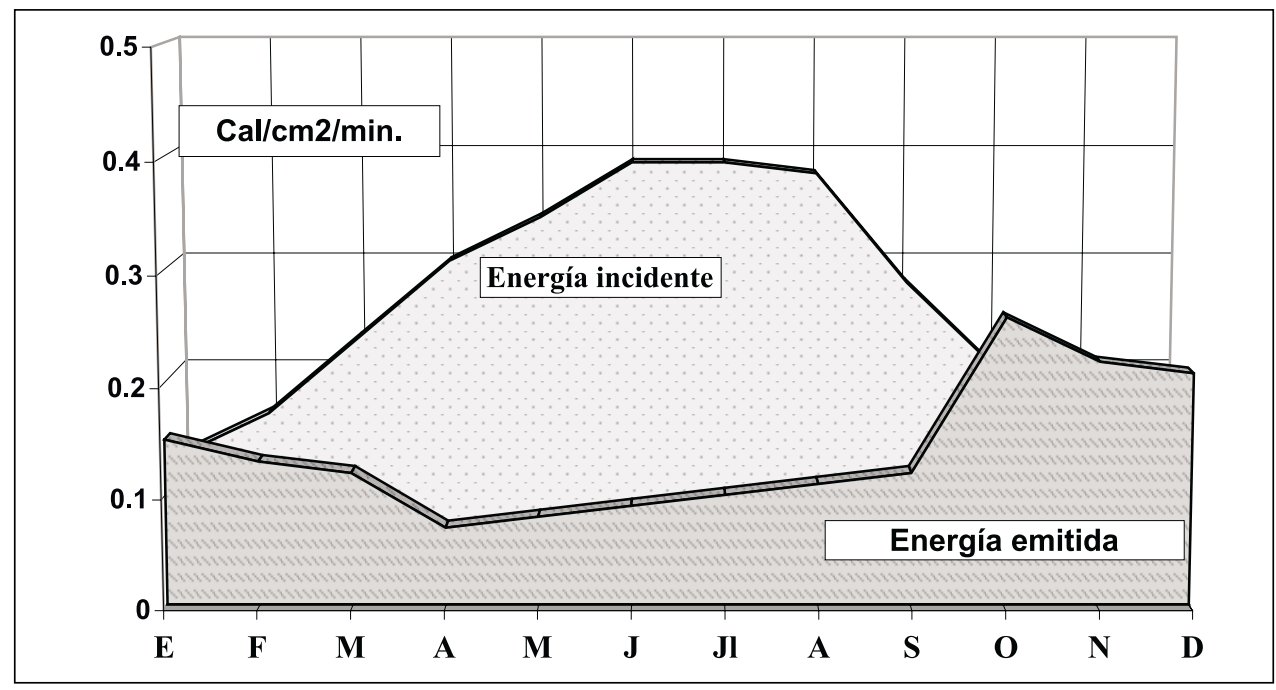

FIGURA 10. Los intercambios energéticos en la interfase atmósfera-mar muestran que las situaciones de estabilidad atmosférica predominan durante la mayor parte del año. Únicamente durante el otoño las fuertes emisiones de calor mar-atmósfera potencian una notable inestabilidad.

La figura muestra que durante una gran parte del año las transferencias energéticas se establecen desde la atmósfera hacia el mar. Ello tiende a estabilizar las situaciones atmosféricas con inhibición de los procesos convectivos. Precisamente esta acumulación de energía es, a su vez, la causa de que durante otra parte del año, especialmente el otoño, la superficie marina potencie una activa convección. La génesis y evolución de las grandes perturbaciones meteorológicas mediterráneas parecen ligadas a la fuerte convección que se desarrolla en los momentos en que advecciones de aire frío irrumpen sobre las aguas cálidas del mar (J. Quereda, 1986). De este modo, el análisis del estado y régimen del campo térmico del mar puede proporcionarnos valiosas informaciones para la previsión de los sucesos meteorológicos.

\section{Convección y situaciones atmosféricas}

Las distintas situaciones atmosféricas, que han regido la evolución climática del año 2001, han constituido una buena expresión de este decisivo papel que las interacciones atmósfera-mar juegan en la climatología de la cuenca occidental del Mediterráneo. Como hemos afirmado, desde el fondo de la cuenca, un mar notablemente cálido durante el otoño e invierno constituye un manantial caliente para la masa atmosférica mientras que en primavera y verano su función refrigeradora se deja sentir estabilizando la atmósfera. Este campo térmico ha vuelto a ser decisivo para unos intercambios energéticos claves en la climatología mediterránea.

En efecto, la mayor parte del año ha estado caracterizada por el predominio de largas situaciones de circulación predominantemente zonal con estabilidad atmosférica, elevadas temperaturas y escasas precipitaciones. Desde el mes de enero hasta el mes de agosto las temperaturas atmosféricas y las de la superficie marina han mostrado valores muy próximos (figura 11). La convección no se ha mostrado activa y las precipitaciones han sido realmente escasas. 


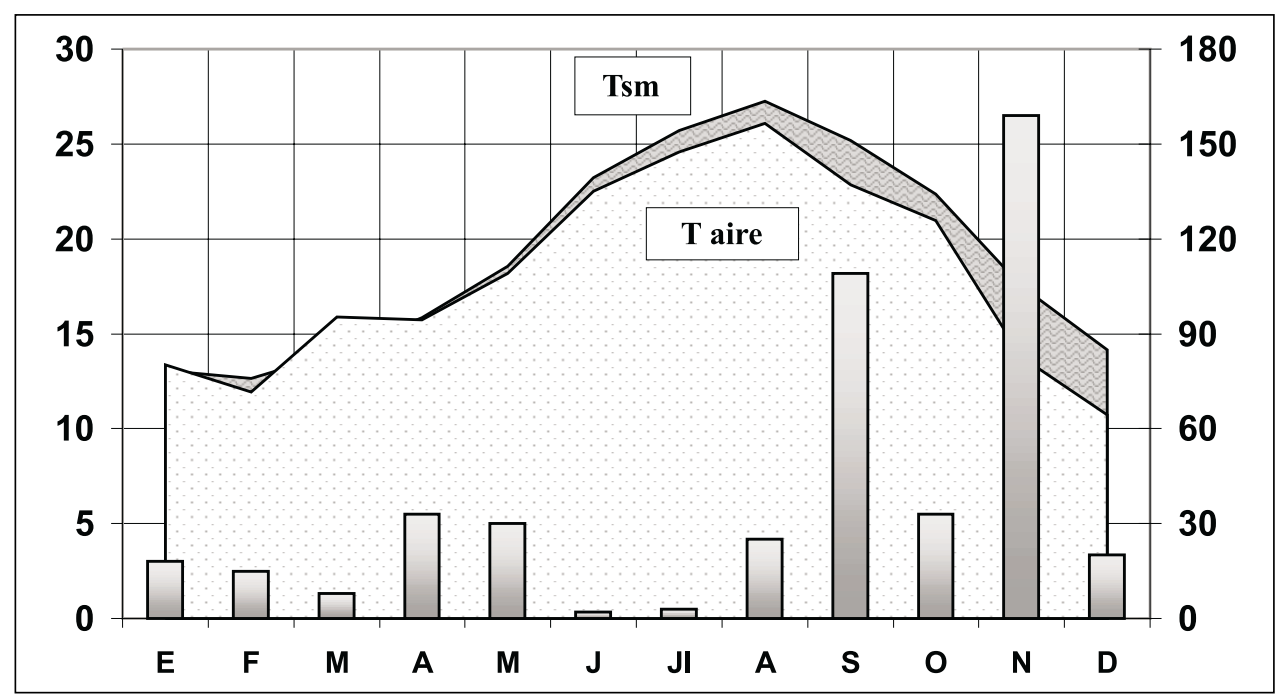

Figura 11. Regímenes de la Temperatura aérea, de la Tsm y de las precipitaciones durante el año 2001 en el observatorio marino.

Esta dinámica atmosférica cambió completamente desde finales de agosto. La energía acumulada durante el verano originó la formación de notables gradientes térmicos entre mar y atmósfera. La Tsm registraba un valor medio de $27^{\circ} \mathrm{C}$ en el mes de agosto y con valores que se incrementan hacia finales del mes en que se sitúan próximos a los $28{ }^{\circ} \mathrm{C}$ (figura 12).

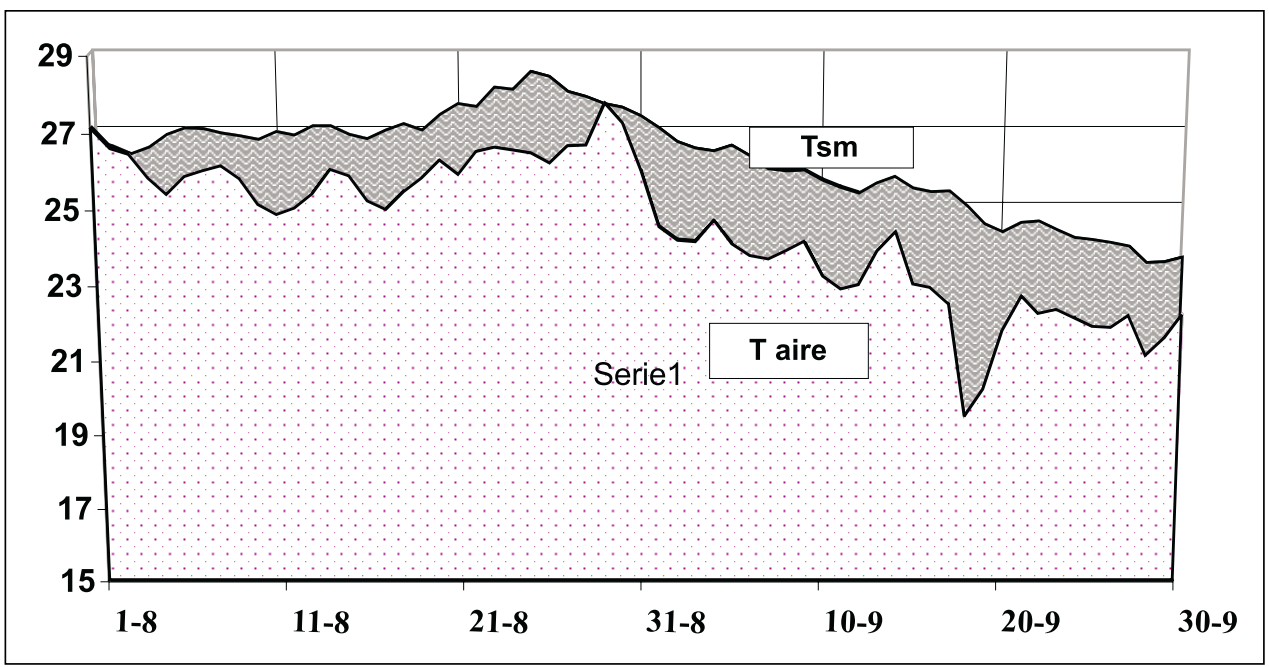

Figura 12. Evolución de la Tsm y de la temperatura del aire durante los meses de agosto y septiembre. Resulta evidente que la acumulación de calor en el mar durante el verano propicia la progresiva exageración de gradientes hacia el otoño. 


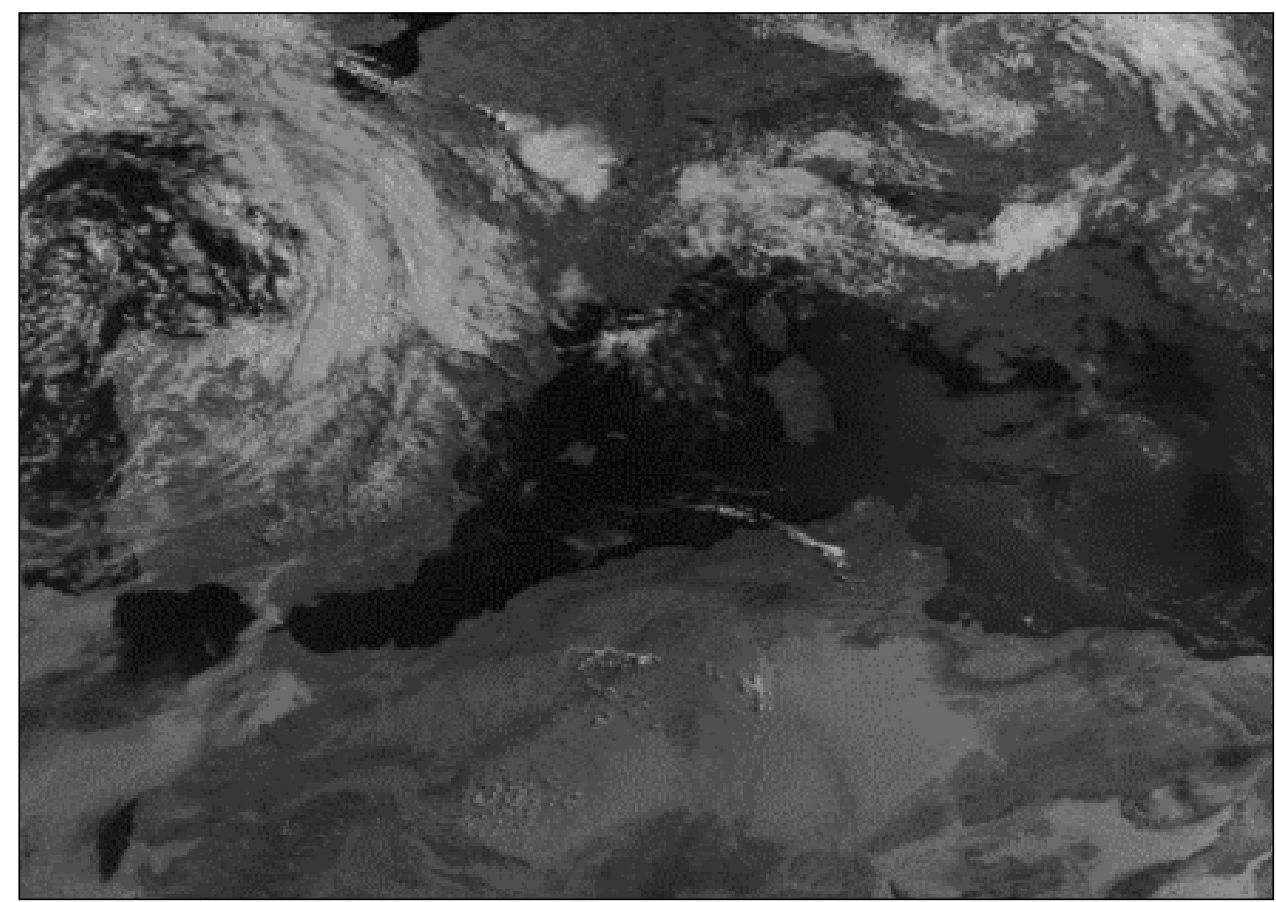

Figura 13. Las circulaciones zonales bajas y en omega marcan la transición hacia las circulaciones meridianas. Imagen NOAA, 20-IX-2001, Universitat Jaume I.

La presencia de esta anomalía reflejaba la acumulación de esos excesos de calor que están en la base de las grandes alteraciones de la circulación general. A través de ellas la atmósfera parece comportarse como un gigantesco condensador que mediante las expulsiones de aire tropical o polar trata de restablecer el equilibrio energético a gran escala, sacando, precisamente la energía de sus reservorios marinos y oceánicos para redistribuirla atmosféricamente. Mecanismo capital en este proceso es la evaporación ya que cada gramo de agua evaporada hace pasar 2500 julios a la atmósfera, es decir, 600 calorías. Para ello, las circulaciones predominantemente zonales adquirieron componentes meridianas. Este drenaje de aire frío polar contribuyó, a su vez, a exagerar los gradientes térmicos así como los procesos de termoconvección (figura 13).

Estos procesos convectivos están en la base de la ciclogénesis sobrevenida en los días 18 y 19 de septiembre. La temperatura superficial del mar con más de $27^{\circ} \mathrm{C}$ a comienzos de mes, se enfría hasta los $23-24^{\circ} \mathrm{C}$ a finales del mismo. En este enfriamiento de las aguas superficiales contribuye decisivamente la intensificación de los procesos evaporativos ligados a la génesis de la perturbación. A medida que la columna de aire frío va aproximándose, la exageración de gradientes térmicos aire-mar provoca el aumento de la velocidad del viento y consecuentemente de la evaporación. La Tsm, cuya amplitud media diaria es de $0.35^{\circ} \mathrm{C}$, desciende más de un grado centígrado en las 24 horas previas al comienzo de las precipitaciones. Posteriormente y cuando la columna ciclogenética se instala sobre el mar, los procesos evaporativos se intensifican y la Tsm se enfría cerca de $3{ }^{\circ} \mathrm{C}$. La evaporación determina que se active una potente convección en el seno de las aguas 


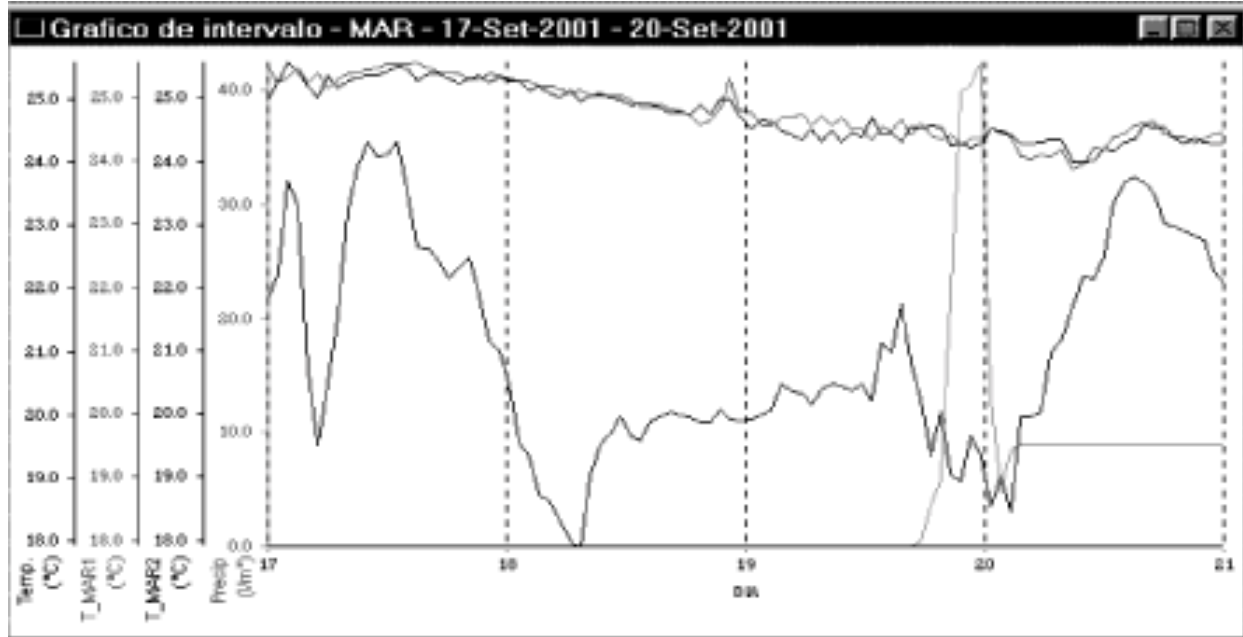

FIGURA 14. Interacciones atmósfera-mar en la ciclogénesis de los días 17 a 20 de septiembre de 2001 (Observatorio Universitario, Plataforma de BPOil). Las sondas de la Tsm, curvas superiores, a -2 y $-3 \mathrm{~m}$, acusan un enfriamiento desde $\operatorname{los} 26^{\circ} \mathrm{C}$ a los $24^{\circ} \mathrm{C}$.

superficiales del mar. A causa de ello, y mientras duran las precipitaciones, la temperatura de la capa más superficial del mar aparece algunas décimas de grado más fría que la de las aguas inmediatamente subyacentes (figura 14).

Idénticos procesos se registraron durante los meses finales del año. La continuada exageración de los gradientes térmicos entre la atmósfera y el mar alcanzaba máximos valores en el mes de noviembre. Las circulaciones zonales bajas, predominantes hasta el mes de octubre, ceden a las circulaciones meridianas. Estos son los mecanismos de la Circulación General de la Atmósfera para equilibrar las anomalías térmicas positivas que todavía registra la Tsm próxima a los $22{ }^{\circ} \mathrm{C}$ a comienzos de noviembre (figura 15).

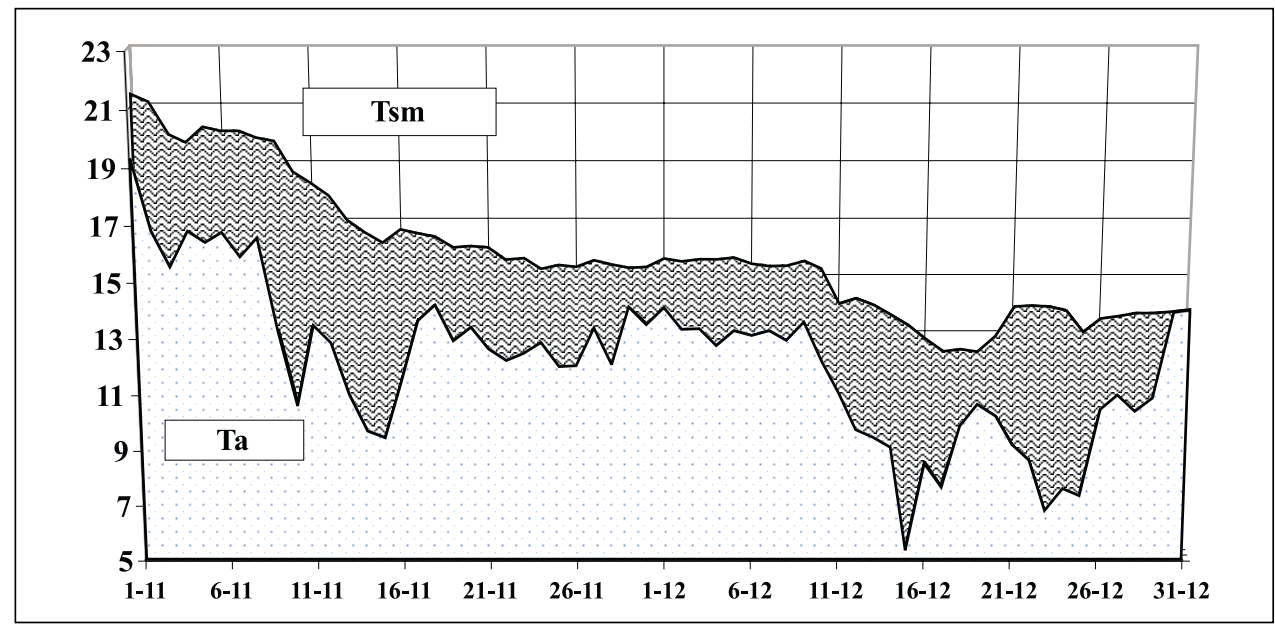

FIgURA 15. Evolución de la Tsm y de la temperatura del aire durante los meses de noviembre y diciembre (Observatorio Universitario, Plataforma de BPOil). 


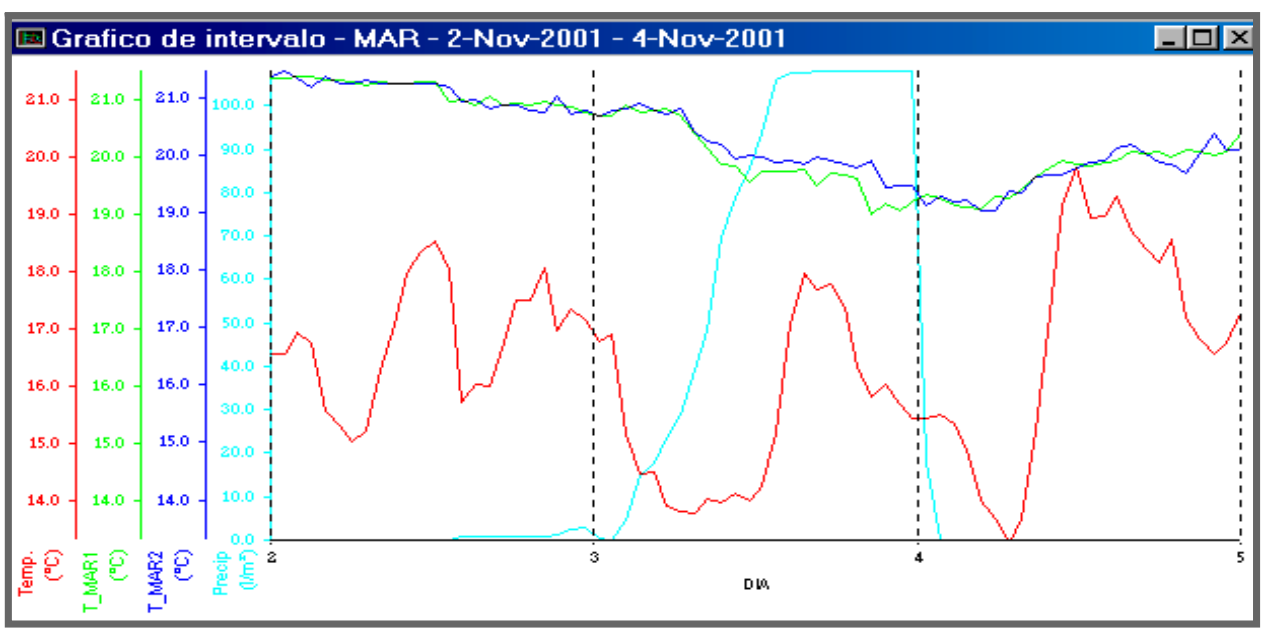

FIGURA 16. Interacciones energéticas atmósfera-mar durante la ciclogénesis del 2 al 4 de noviembre de 2001 (Observatorio Universitario, Plataforma de BPOil).

Estos mecanismos se manifestaron en la gran ciclogénesis acontecida entre los días 2 y 4 de noviembre, con precipitaciones superiores a los 100 litros $\mathrm{m}^{-2}$. La superficie marina se muestra notablemente cálida con $22^{\circ} \mathrm{C}$, determinando unos gradientes aire-mar próximos a $\operatorname{los} 6{ }^{\circ} \mathrm{C}$. Las cuantiosas e intensas precipitaciones registradas materializan todos los procesos convectivos y de transferencia energética desarrollados en la interfase atmósferamar (figura 16).

De este modo, resulta evidente que la exageración de los gradientes térmicos aire-agua genera flujos de calor sensible y latente que disparan el calor y la humedad en el seno de las formaciones nubosas. Unos procesos que se intensifican cuando las expulsiones de aire frío se superponen sobre las aguas cálidas del Mediterráneo. Se puede observar entonces la formación de numerosos cumulonimbos que se van reagrupando hasta dar, 24 horas después del comienzo de su aparición, un sistema nuboso de forma aproximadamente circular y cuyo diámetro es del orden de $800 \mathrm{Km}$ en media (figura 17).

Incluso en numerosas ocasiones parece que la presencia de numerosos cumulonimbos en torno al centro de la espiral depresionaria, juegue un importante papel en la activación de las depresiones de altitud por enfriamiento de la troposfera media y superior (R. M. Thepenier, 1987). Consecuentemente con ello los mapas de altura muestran que la aparición del torbellino coincide con la de un mínimo de temperatura, materializado por isotermas horizontales de forma aproximadamente circular y cuyo centro corresponde al núcleo del torbellino. Este mínimo de temperatura se observa en toda la porción de la troposfera comprendida entre los niveles de 700 y $300 \mathrm{hPa}$ (figura 18).

En este orden de observaciones, y en particular la presencia de ese mínimo de temperatura en los niveles de 700 a $300 \mathrm{hPa}$ asociado al torbellino ciclónico, permiten pensar que los cumulonimbos de estos sistemas nubosos pueden jugar un activo papel en la evolución de los mismos (R. M. Thepenier et D. Cruette, 1977). No en balde en el seno de esos cumulonimbos se operan importantes intercambios de energía en la continua transformación de estado del vapor mediante las corrientes verticales de ascendencia y subsidencia. De ahí que los cumulonimbos puedan ser considerados como fuentes de calor en la 


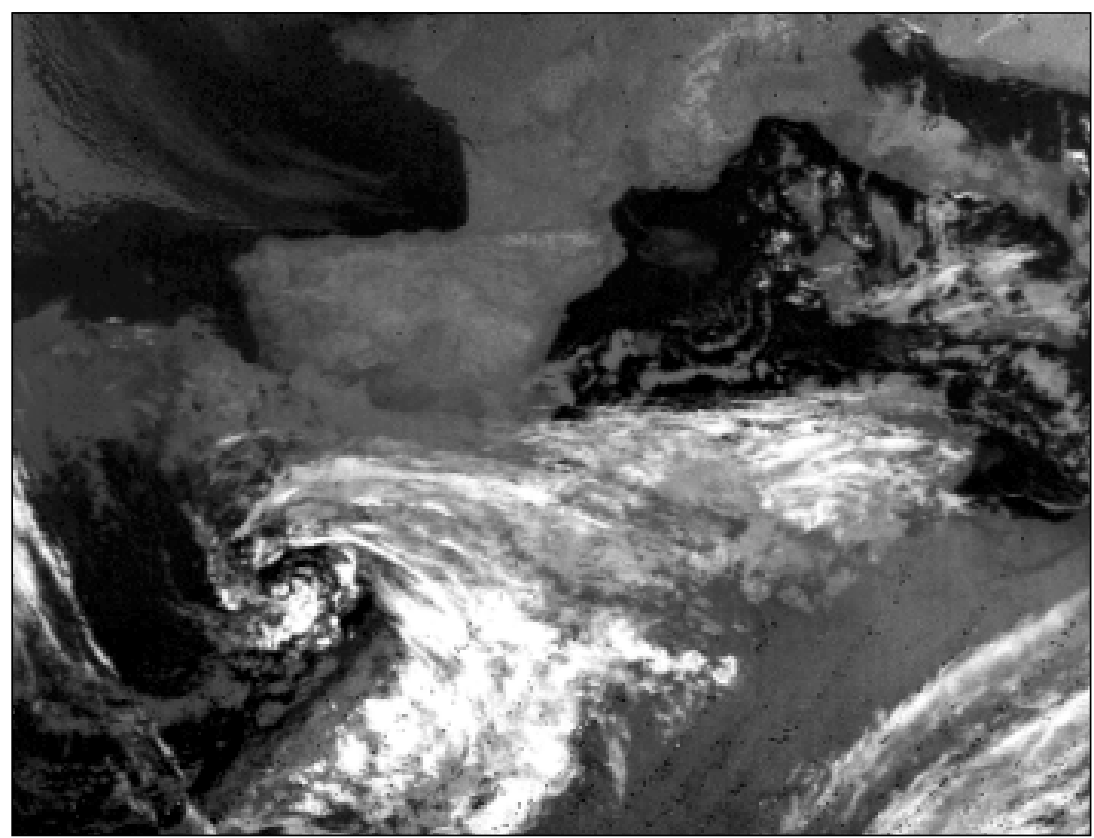

FIGURA 17. Torbellino de cumulonimbos convectivos sobre Gibraltar mostrando las bajas temperaturas en la brillantez de sus cimas (Imagen NOAA-12, 12-XII-2001, Universitat Jaume I).

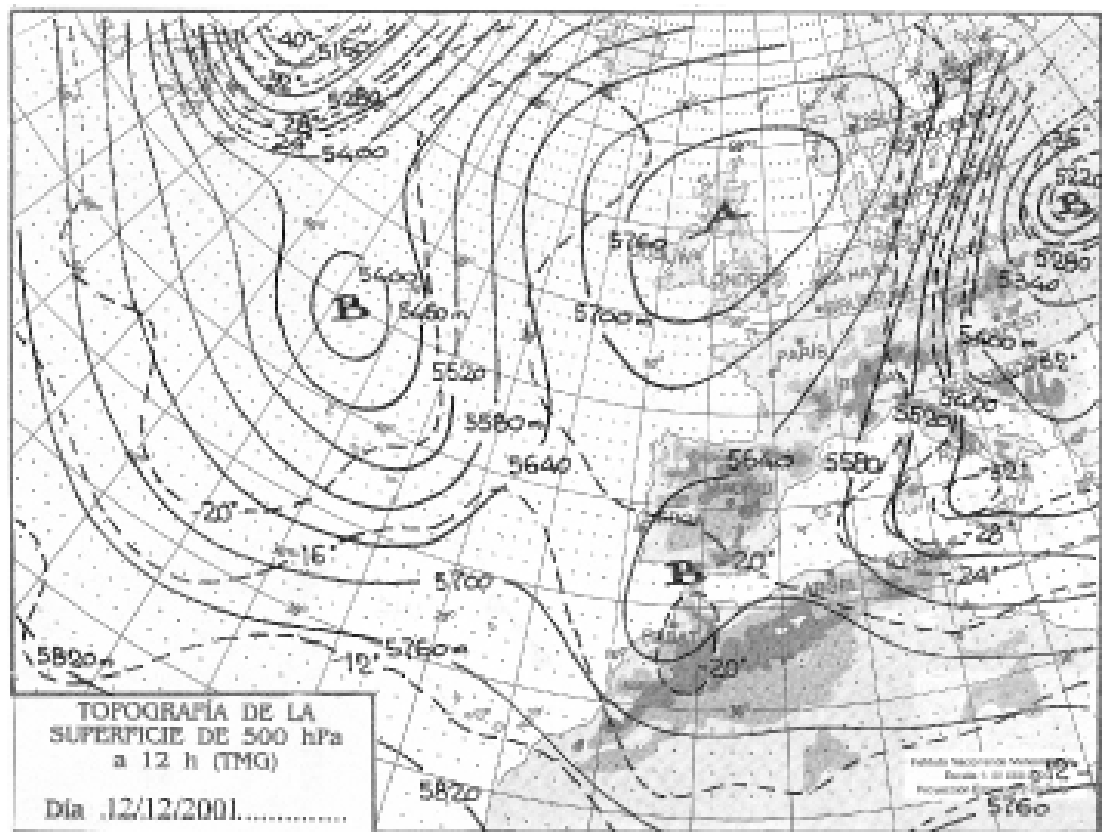

FIGURA 18. Topografía de $500 \mathrm{hPa}$ a las 12 horas del 12-XII-2001 (INM). La progresiva aparición e intensificación en altura del mínimo térmico en el centro del conjunto convectivo parece sugerir el activo papel del sistema nuboso en la profundización del torbellino depresionario. 
troposfera inferior o media (calor latente liberado por la condensación del vapor de agua) y como fuente fría en la troposfera superior (P. Queney, 1974). De este modo y consecuentemente con el hecho de que la temperatura media en el interior del sistema nuboso sea inferior a la del aire ambiente a escala sinóptica, se potencia, en virtud de la ley hidrostática, que el decrecimiento vertical de la presión en el seno del sistema nuboso será más fuerte que el que pueda producirse en el exterior del sistema. Déficit que se va acentuando hasta los $300 \mathrm{hPa}$.

\section{Conclusiones}

Las observaciones meteorológicas efectuadas a lo largo del año 2001 en el observatorio marino de la Universitat Jaume I, han permitido verificar que la cuenca occidental del Mediterráneo constituye una zona neurálgica por lo que concierne a las interacciones atmósfera-mar. Las distintas situaciones atmosféricas observadas durante este año han mostrado que tanto las largas situaciones de estabilidad atmosférica como especialmente la génesis y evolución de sus grandes perturbaciones meteorológicas han venido determinadas por el desarrollo más o menos intenso de los procesos de termoconvección que se han generado en el interior de esa cubeta marina. Es más, las observaciones efectuadas a lo largo del año 2001, permiten interrogarnos sobre si tanto el mismo drenaje del aire frío hacia nuestro ámbito como la misma intensificación de los torbellinos ciclogenéticos podrían estar siendo activados por las anomalías registradas en el campo térmico del mar y su papel convectivo.

\section{Bibliografía}

BROCHET, P. et GERBIER, N. (1975): L'evapotranspiration. Aspect agrométéorologique évaluation pratique de l'évapatranspiration potentielle, S.M.M. Climatologie, Paris, 95 pp.

JANSA GUARDIOLA, J. M. (1966): «La Meteorología en el Mediterráneo Occidental», Tercer Ciclo de Conferencias, I.N.M. 1964, pp. 1-35.

NAYA, J. A. (1984): Meteorología superior. Espasa-Calpe, Madrid, 546 pp.

PERRIN de BRICHAMBAULT, Ch. (1978): «Estimation de l'énergie solaire disponible au sol». La Météorologie, VI, 15, pp. 5-45.

QUENEY, P. (1974): Elements de Météorologie. Masson, Paris, 320 pp.

QUEREDA, J. y CALVO, A. (1981): «El ciclo del agua y su importancia». Millars, VII, pp. 25-48.

QUEREDA, J. (1982): Castellón: precipitaciones y radiación solar, Ayto. Castellón, 39 pp.

QUEREDA, J. (1986): «Un aspect du regime thermique en Mediterranée Occidentale». Met-mar, 131, pp. 13-18.

QUEREDA, J. y MONTÓN, E. (1994): Las lluvias torrenciales en la Comunidad Valenciana. Interacciones atmósfera-mar. Dip. de Castelló, $190 \mathrm{pp}$.

QUEREDA, J. et al., (2001): Nuestro porvenir climático ¿Un escenario de aridez?, Universitat Jaume, I, $223 \mathrm{pp}$.

RESNYANSKII, Y. D. (1985): Indirect estimate of thickness of mixed layer according to data on ocean surface temperature», Soviet Meteorology and Hidrology, 11, pp. 38-46.

SINOLECKA, C. et HONTARRÈDE, M. (2000): «Rayonnement solaire et albédo», Met-Mar, 188, pp. 3-5.

SUKHOVEI, V. F. and SAID, M. A. M. (1985): «Thermal interaction of the Mediterranean Sea and the Atmosphere». Meteorologiya i Gidrologiya, 9, pp. 55-62.

THEPENIER, R. M. (1987): «Role de la convection orageuse dans l'evolution des tourbillons cycloniques sur l'Europe occidental», C.R. Acad., 282, Série B, pp. 251-257.

THEPENIER, R. M. et CRUETTE, D. (1977): «Role des cumulonimbus dans l'evolution des perturbations cycliques des regions mediterranénnes», La Météorologie, pp. 165-171. 